\title{
Simulation of YBCO Tape and Coils in HTS Maglev System
}

\author{
Mengxiao Song and Jie Li \\ College of Mechatronic Engineering and Automation, National University of Defense Technology, Changsha 410073, P. R. China
}

\begin{abstract}
In the process of running high temperature superconducting maglev train, the AC(Alternating Current) loss of superconducting coil is directly related to its safe operation and operating cost. In this paper, the simulation model was built based on the finite element software COMSOL Multiphysics, and mainly simulated and calculated the AC losses of YBCO(Yttrium Barium Copper Oxide) tape and coils. In this model, as the solving object, the singular and infinite long YBCO tape and coils model was solved with $\mathrm{H}$-formulation and the nonlinear characteristic (E-J constitutive law) and anisotrophy (B-J characteristic) were taken into consideration as the theoretical foundation. Then on the basis of the model under maglev suspension system, AC losses under different amplitude and frequence $\mathrm{AC}$ currents were calculated. The results shows that under different frequencies and dynamic components, the local maximum AC loss of YBCO tape and coils occurs when the steady-state DC(Direct Current) current is 30A. Then comparing with old maglev suspension system, the new system can greatly reduce the energy consumption and the material cost.
\end{abstract}

\section{Introduction}

Maglev is considered a promising way of rail transport system. It has lots of advantages which traditional wheelrail trains do not have. Maglev trains does not contact tracks during running, so that there is no mechanical friction, which makes the noise greatly reduced. It is generally driven by linear motor, so it has great ability at climbing and small turning radius. In addition, the maglev can construct railways through viaduct, so there is less impact on the surrounding environment and lower cost [1-2].

Currently maglev train uses aluminum coils, so there are resistive losses in the suspension process which makes the electromagnets generate more heat, but also results in a waste of energy. Currently power consumption of suspension system is 0.75 kilowatts per ton. If the resistive losses can be reduced, the maglev train will save more energy[3-4].

Superconducting material can significantly reduce the resistance loss of the conventional normal conducting coils. High-temperature superconducting material is a kind of material working in the liquid nitrogen which lowers the requirements of refrigeration and insulation. High-temperature superconducting maglev is becoming the trend of future transportation development [5-6].

Research on AC loss of high-temperature superconducting maglev, obtaining loss characteristics, so we can design the HTS(High-Temperature Superconductor) suspension electromagnet, what's more, the thermal insulation structure and the power of refrigerator etc. Ultimately, assess the possibility of applying the superconducting material on electromagnetic levitation. So the primary issue of applying high-temperature superconducting material in maglev system is to study the magnitude of AC loss[7$10]$.

This paper is based on the E-J constitutive law of YBCO and the finite element software COMSOL Multiphysics. Mainly simulate and calculate the AC losses of YBCO tape and coils under different kinds of current inputs. The results shows that under different frequencies and dynamic components, the local maximum AC losses of YBCO tape and coils occurs when the steady-state DC current is $30 \mathrm{~A}[11-13]$.

\section{Simulation}

Fig. 1 and Fig. 3 show the simulation of YBCO tape model and coils model respectively, model parameters and expressions shown in Table 1 and Table 2.

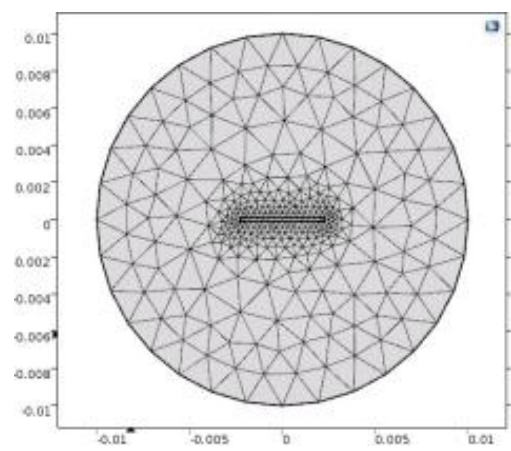

Figure 1. simulation of YBCO tape model 


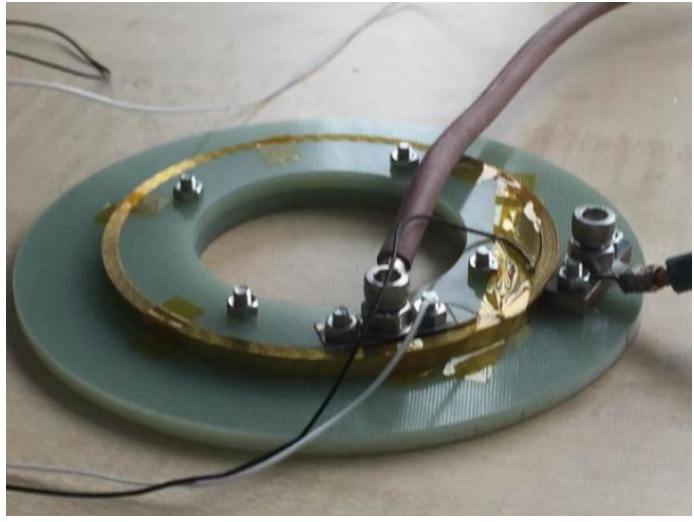

Figure 2. the photo of YBCO coils

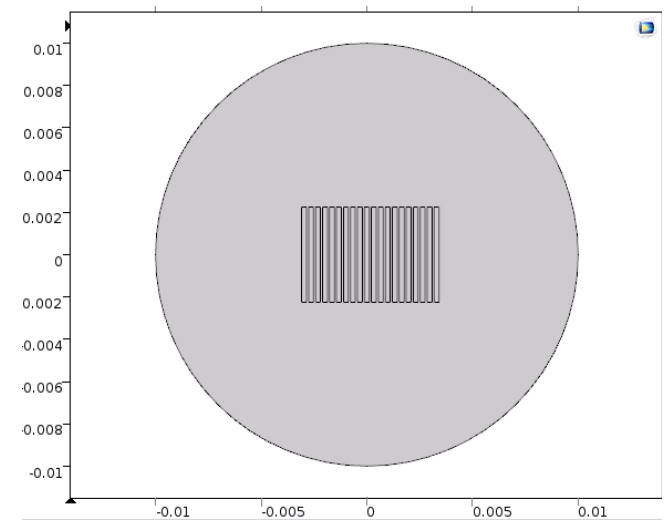

Figure 3. simulation of YBCO coils model

Table 1. Primary parameters

\begin{tabular}{|l|l|}
\hline tape width & $4.5 \mathrm{~mm}$ \\
\hline tape thickness & $0.23 \mathrm{~mm}$ \\
\hline critical current & $120 \mathrm{~A}$ \\
\hline critical current density & $1.17 \mathrm{e} 8 \mathrm{~A} / \mathrm{m}^{\wedge} 2$ \\
\hline simulation time & $0.1 \mathrm{~s}$ \\
\hline distance between turns & $0.1 \mathrm{~mm}$ \\
\hline
\end{tabular}

Table 2. Primary variables and expressions

\begin{tabular}{|c|c|c|}
\hline variables & expressions & description \\
\hline$J$ & $\frac{\partial H_{y}}{\partial x}-\frac{\partial H_{x}}{\partial y}$ & $\begin{array}{l}\text { Current density } \\
\qquad\left(\mathrm{A} / \mathrm{m}^{\wedge} 2\right)\end{array}$ \\
\hline $\boldsymbol{E}$ & $\begin{cases}0 & J<J \\
\left\{\left(\frac{J-J_{\mathrm{r}}}{J_{\mathrm{r}}}\right)\right. & J \geq J_{\mathrm{r}}\end{cases}$ & $\begin{array}{l}\text { Electric field strength } \\
(\mathrm{V} / \mathrm{m}) \\
\text { E-J constitutive law }\end{array}$ \\
\hline$Q$ & $E \times \boldsymbol{J}$ & $\mathrm{AC}$ loss $(\mathrm{W} / \mathrm{m})$ \\
\hline $\boldsymbol{I}$ & $I_{d}+I_{0} \sin (1, t)$ & current applied (A) \\
\hline
\end{tabular}

This simulation is based on different types of given current to calculate the AC losses of the tape and coils.

\section{Results and analysis}

\subsection{The tape simulation}

Current given is

$$
I=I_{d}+I_{\alpha} \sin (2 \pi f t)
$$

AC losses under various parameters are shown below. Among them $I_{d}$ changes from $10 \mathrm{~A}$ to $70 \mathrm{~A}$ with the step of 10A. $I_{\alpha}$ changes from 5A to 20A with the step of 5A $f$ changes from $10 \mathrm{~Hz}$ to $500 \mathrm{~Hz}$.

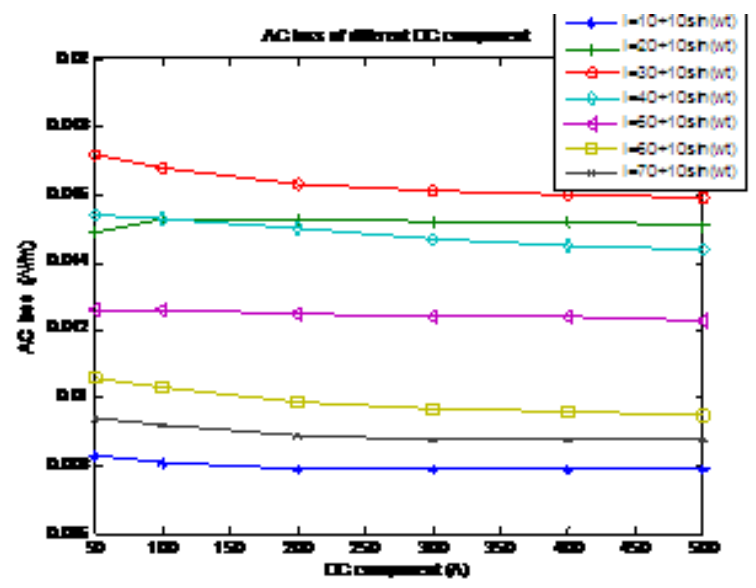

Figure 4. $\mathrm{AC}$ losses of different $\mathrm{DC}$ components under different frequencies

As can be seen from Fig. 4, with $I_{\alpha}$ being 10A, the change of frequency makes no significant effect on AC losses in different

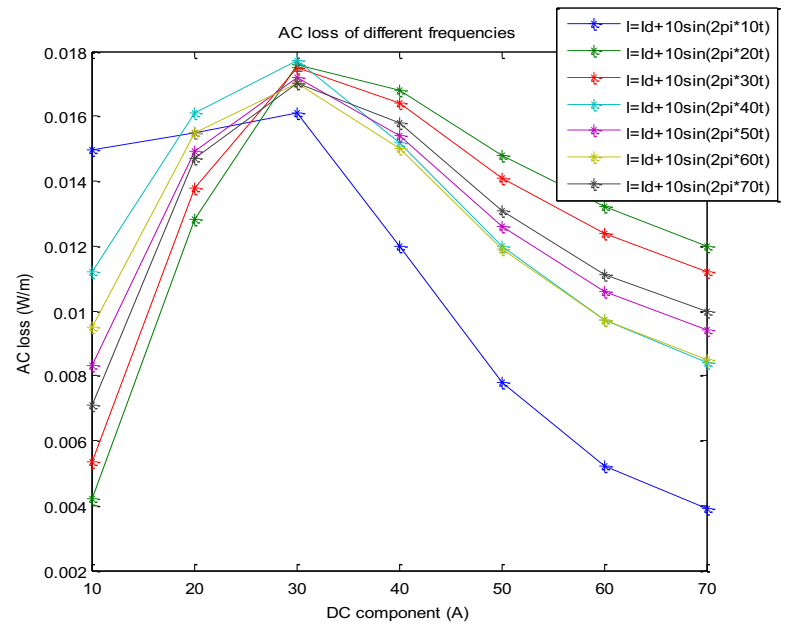

Figure 5. AC losses of $10 \sim 70 \mathrm{~Hz}$ under different DC components 


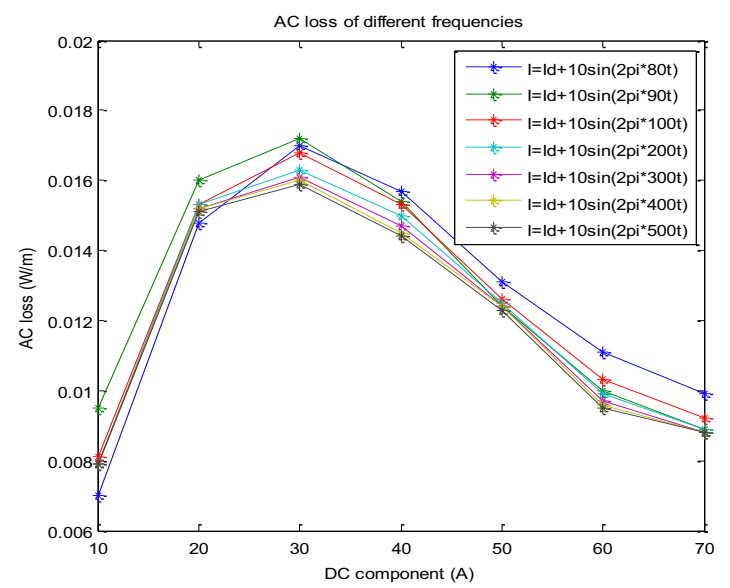

Figure 6. AC losses of $80 \sim 500 \mathrm{~Hz}$ under different DC components

As can be seen from Fig. 5 and Fig. 6, with $I_{a}$ being $10 \mathrm{~A}$, at each frequency, the $\mathrm{AC}$ loss occurs local maximum when the DC component is $30 \mathrm{~A}$. Thus AC losses can be reduced if avoiding $30 \mathrm{~A}$ and its neighborhood.

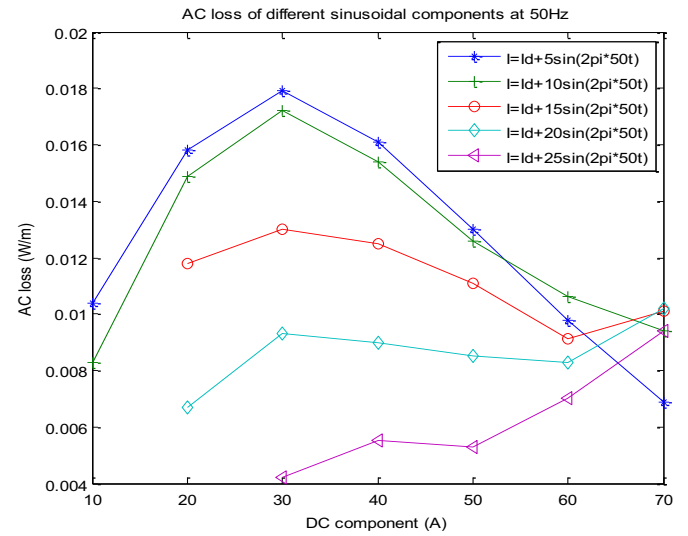

Figure 7. AC losses of different sinusoidal components under different DC components at $50 \mathrm{~Hz}$

As can be seen from Figure 7, at $50 \mathrm{~Hz}$, for different sinusoidal components, the AC loss occurs local maximum when the DC component is 30A. Although there is a rally after $60 \mathrm{~A}, \mathrm{AC}$ losses can be reduced if avoiding $30 \mathrm{~A}$ and its neighborhood.

\subsection{The coils}

From above, select a set of representative parameters to calculate AC loss of YBCO coils model.

Current given is $I=I_{d}+I_{a} \sin (2 \pi f t)$

AC losses under various parameters are shown below. Among them $I_{d}$ changes from $10 \mathrm{~A}$ to $70 \mathrm{~A}$ with the step of $10 \mathrm{~A}$. $I_{a}=5 \mathrm{~A}$ and $f=20 \mathrm{~Hz}$.

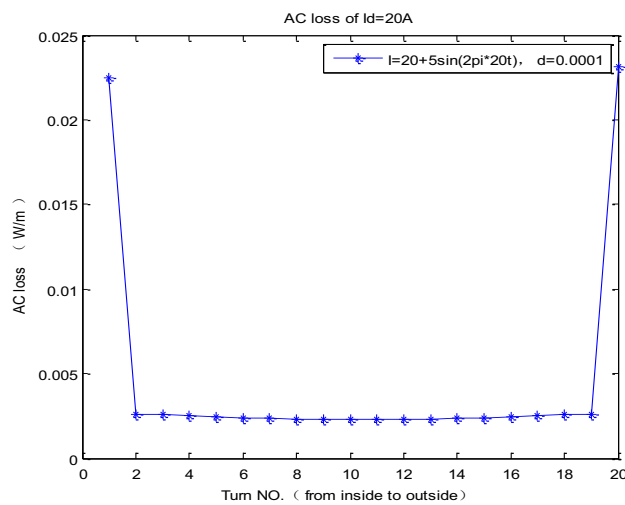

(a)

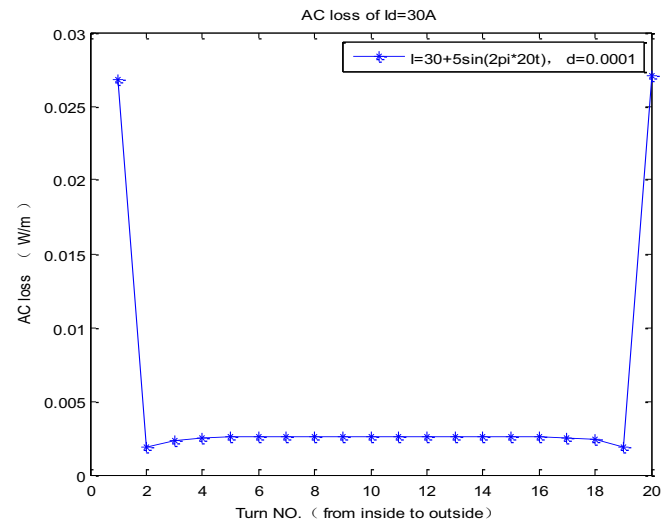

(b)

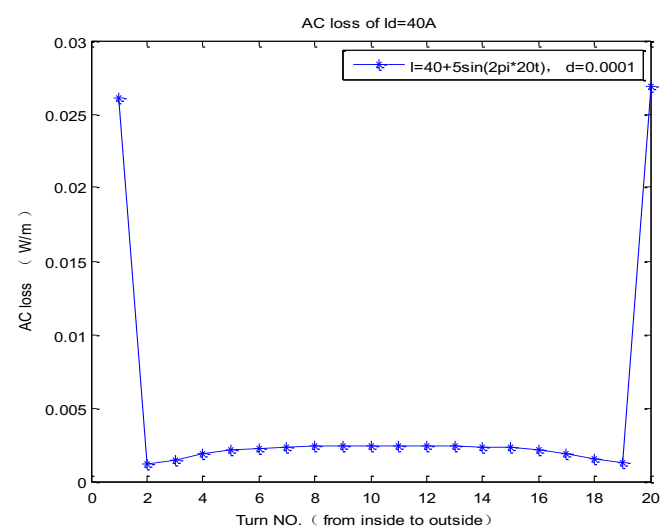

(c)

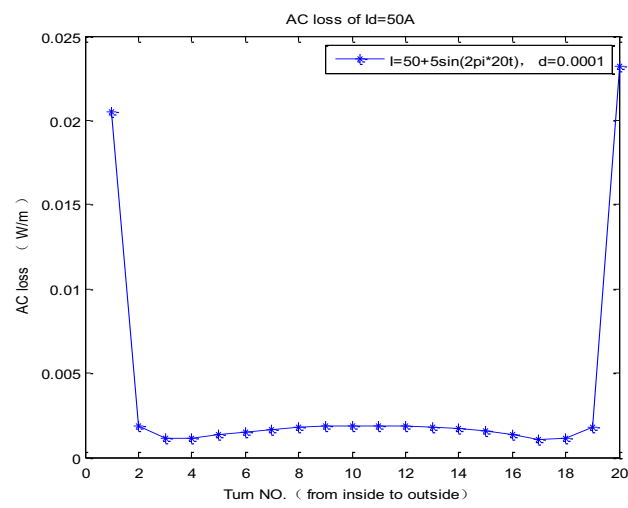

(d) 


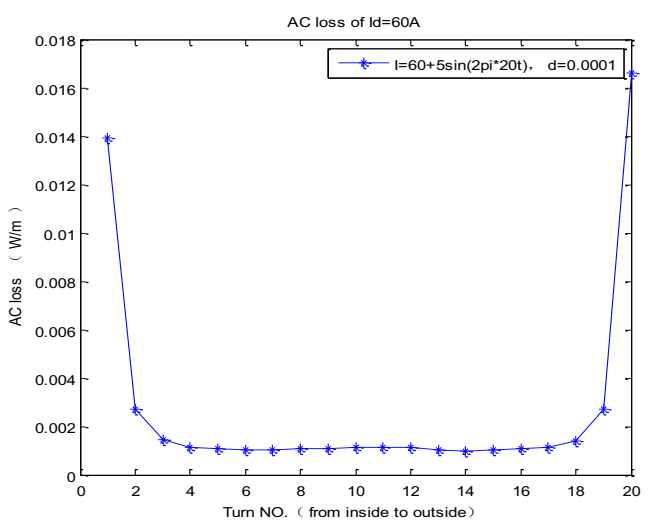

(e)

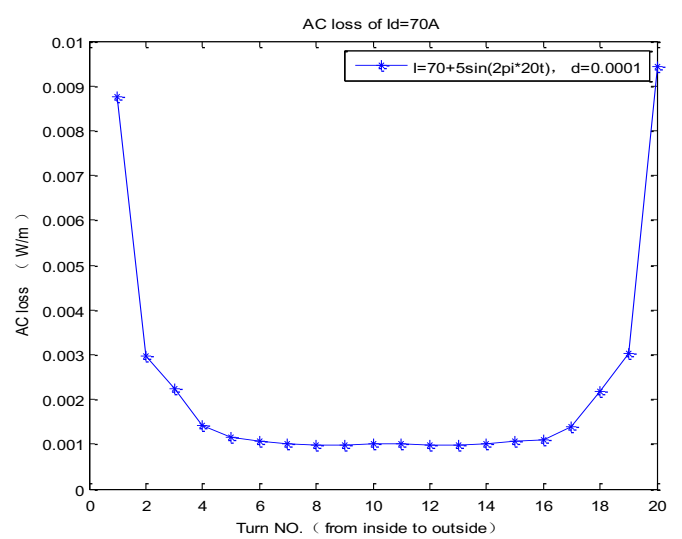

(f)

Figures 8. (a f) AC losses of $20 \mathrm{~Hz}$ and $I_{a}=5 \mathrm{~A}$ under different DC components from $20 \mathrm{~A}$ to $70 \mathrm{~A}$

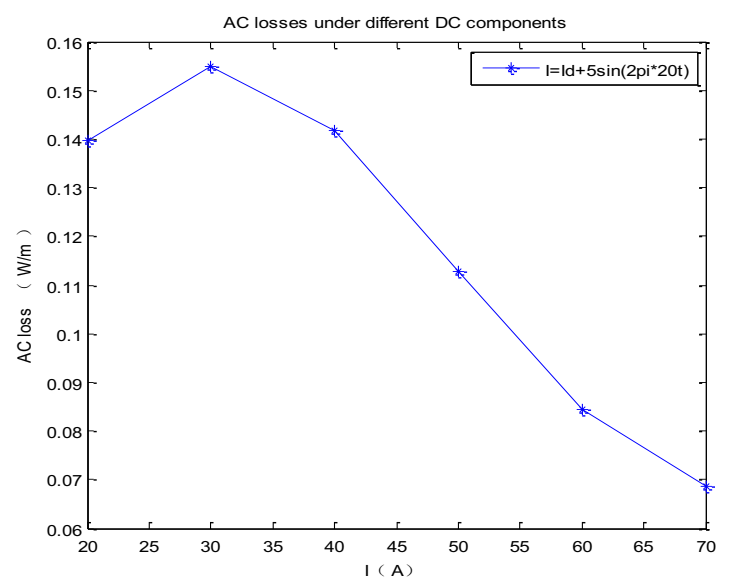

Figure 9. normalized AC losses under different DC components

This normalization means multiplying the corresponding length of every turn of the coils then add them all to obtain the total AC loss.

As can be seen from the simulation above, with $I_{a}$ being 10A, $f$ being $20 \mathrm{~Hz}$, the AC loss occurs local maximum when the DC component is $30 \mathrm{~A}$. This result is the same as the simulation of YBCO tape.

\section{Conclusion}

Equations should be centred and should be numbered with the number on the right-hand side.As for maglev system, saving the cost and reducing energy consumption have always been the objectives to pursue.

Based on the above conclusions, if we improve the DC components of work current from $30 \mathrm{~A}$ to $50 \mathrm{~A}$, the AC loss will drop from $0.0172 \mathrm{~W} / \mathrm{m}$ to $0.0126 \mathrm{~W} / \mathrm{m}$, a decrease of $26.74 \%$. Meanwhile, with the same magnetic motive force, the coil turns required will reduce by $40 \%$. What' $\mathrm{s}$ more, the total reduction of cost will be more than $40 \%$ due to the winding accumulation and the low success rate of winding coils. For example, when the DC component of current input is $30 \mathrm{~A}$, we need about $400 \mathrm{~m}$ tape with the market price about 400 yuan / $\mathrm{m}$. Therefore, this improvement will bring very substantial cost savings. Then we turn back to see the energy consumption.

$$
E_{\text {new }}=(1-26.74 \%) \times(1-40 \%) E_{\text {old }}=44.0 \% E_{\text {old }}
$$

This improvement will reduce the energy consumption by more than $50 \%$. The new one is only $44 \%$ of the old one.

The above conclusions are on the contrary to the traditional cognition that the greater the current is, the greater the AC loss will be. The author was very surprised when firstly found this conclusion, after constantly repeating and verifying this simulation, still got this conclusion. Finally decide to propose this result by this paper to have a discussion with the readers.

\section{References}

1. Yan L G. IEEE Transactions on Applied Superconductivity, 2006, 16(2):1138-1141.

2. Yan L G. IEEE Transactions on Applied Superconductivity, 2008, 18(2), 92-99.

3. Salih E, Lachowicz S, Bass O, et al. International Conference on Green Energy. 2014:267-272.

4. Higashikawa K, Nakamura T, Hoshino T. Physica C Superconductivity \& Its Applications, 2005, 419(s 34):129-140.

5. Zhang M, Kvitkovic J, Kim C H, et al. Journal of Applied Physics, 2013, 114(4):043901-043901-8.

6. Yuan W, Campbell A M, Hong Z, et al. Superconductor Science \& Technology, 2010, 23(8):32-32.

7. Zhang, Min. Study of second-generation hightemperature superconducting coils[J]. 2013(October).

8. Zermeno V M R, Abrahamsen A B, Mijatovic N, et al. Journal of Applied Physics, 2013, 114(17):173901-173901-9.

9. Nguyen D N, Sastry P V P S S, Schwartz J. Journal of Applied Physics, 2007, 101(5):053905-053905-9.

10. Grilli F, Ashworth S P. Superconductor Science \& Technology, 2007, 20(8):794-799.

11. Ritesh Kumar and Amit Kumar, International Journal of Mechanical Engineering and Robotics Research, Vol. 3, No. 1, pp. 42-49, January 2014.

12. Boyd A. Nicholds and John P. T. Mo, International Journal of Mechanical Engineering and Robotics Research, Vol. 5, No. 2, pp. 138-143, April 2016. DOI: $10.18178 /$ ijmerr.5.2.138-143 
13. Ngoctan Tran, Yaw-Jen Chang, Jyh-tong Teng, and Thanhtrung Dang, International Journal of Mechanical Engineering and Robotics Research,
Vol.4, No. 3, pp. 204-208, July 2015. DOI: 10.18178/ijmerr.4.3.204-208 